PART III

RELIC RADIATION

(Chairman: M.J.Rees) 


\title{
ENERGY DENSITY OF THE RELIC RADIATION*
}

\author{
A. G. BLAIR
}

Los Alamos Scientific Laboratory, University of California, Los Alamos, N.M., U.S.A.

\begin{abstract}
Experimental measurements of the intensity of the submillimeter background are reviewed. The latest results all indicate a low background; it is not yet possible to say whether or not the spectrum in this range is blackbody.
\end{abstract}

The history of experimental measurements of the energy density of the submillimeter background has been the sort that tries the patience of strong men, whether they are in the scientific community or in the funding agencies. The purpose of my talk is to outline the essential features of these experiments and their results, and to give you my view of where the matter stands at present.

When, through the work of Penzias and Wilson (1965) and Dicke et al. (1965), the notion that the Universe may be bathed in the redshifted remnant of the primordial fireball took firm root in the minds and hearts of many people, it was clear that complete verification awaited measurements in the millimeter and submillimeter wavelength range. The main obstacle to the solution of this problem was, and continues to be, the very great experimental difficulty in making the required measurements. True, groundbased radiometer measurements as short as $3.3 \mathrm{~mm}$ were made by Boynton et al. (1968) and later by Millea et al. (1971), measurements that complied with the requirements of the Planck curve, falling well below the Rayleigh-Jeans line of Figure 1. Also, it was possible to obtain short wavelength brightnesses, or, in most

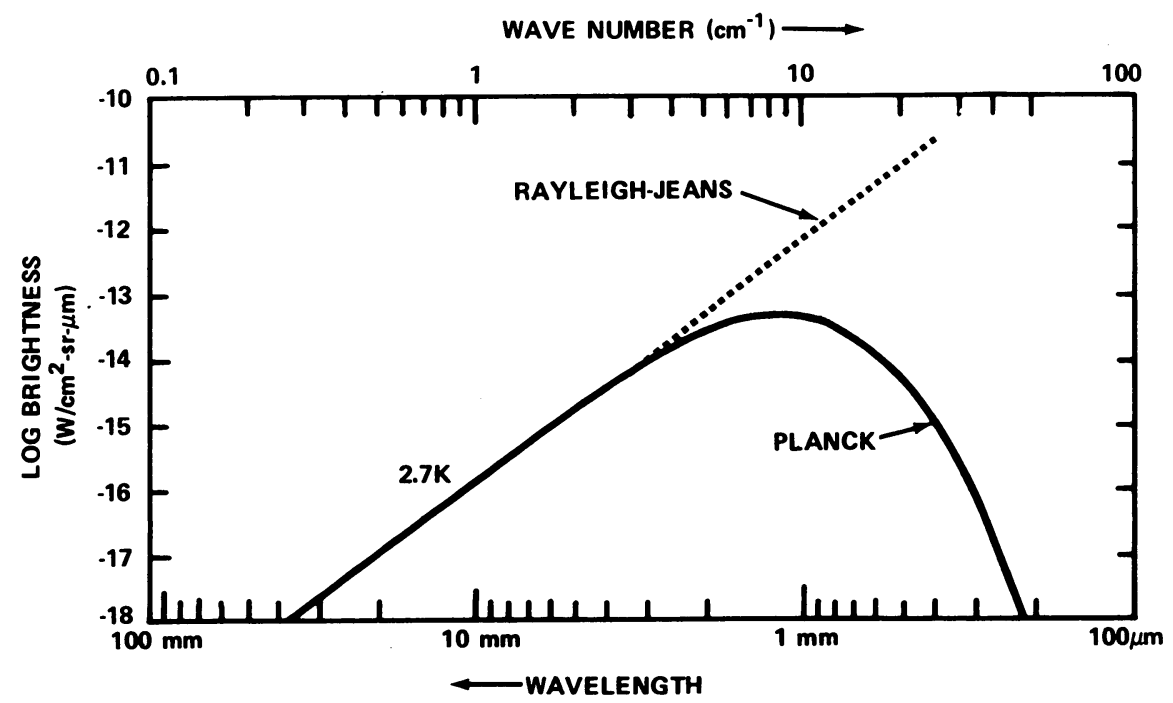

Fig. 1. Brightness curves for $2.7 \mathrm{~K}$ radiation.

* Work performed under the auspices of the U.S. Atomic Energy Commission. 
cases, brightness upper limits, by the indirect method of observing optical absorption of the light of certain well-situated stars (Thaddeus, 1972). It was also possible to obtain upper limits to this short wavelength flux by working backwards, as it were, from measurements of cosmic X-ray and electron backgrounds (Apparao, 1968).

But to attack the problem of direct absolute flux measurements of the cosmic background radiation required conducting the experiments above most of the Earth's atmosphere, for, from about $3 \mathrm{~mm}$, over the $2.7 \mathrm{~K}$ Planck peak at about $1.5 \mathrm{~mm}$, and down the short wavelength side of the Planck curve, the absorption and emission characteristics of the atmosphere are fatal to any such attempt at low altitudes. There have been, however, searches for emission lines in this spectral region, conducted at mountain top sites or on aircraft platforms. I shall return briefly to these measurements later in this paper.

To put into perspective the magnitude of the energy density we are talking about if the Universe really is pervaded by this $2.7 \mathrm{~K}$ blackbody radiation, I show a table (Table I), based on one in an article by Cowsik and Price (1971) that.appeared a couple of years ago in Physics Today. Although one can argue about some of the values

TABLE I

Energy density magnitudes

\begin{tabular}{lcclc}
\hline Region & \multicolumn{2}{l}{ Energy density $\left(\mathrm{eV} \mathrm{cm}^{-3}\right)$} & \\
\cline { 2 - 5 } & Magnetic & Starlight & $\begin{array}{l}2.7 \mathrm{~K} \text { Blackbody } \\
\text { radiation }\end{array}$ & $\begin{array}{l}\text { Cosmic } \\
\text { rays }\end{array}$ \\
\hline Solar system & 100 & $10^{7}$ & 0.25 & $i$ \\
Galaxy (disc) & 1 & 0.5 & 0.25 & 1 \\
Galaxy (halo) & $?$ & 0.1 & 0.25 & $<1 ?$ \\
Universe & $<4 \times 10^{-4}$ & 0.003 & 0.25 & $<10^{-3}$ \\
\hline
\end{tabular}

shown, the point I wish to make here is that the $0.25 \mathrm{eV} \mathrm{cm}^{-3}$ energy density of a $2.7 \mathrm{~K}$ fireball remnant is a rather large number in comparison to the other universal energy densities in the table. Expressed in other units, $\frac{1}{4} \mathrm{eV} \mathrm{cm}^{-3}$ is about $10^{-10} \mathrm{~W} \mathrm{~cm}^{-2} \mathrm{sr}^{-1}$, or in equivalent mass, about $3 \times 10^{-34} \mathrm{~g} \mathrm{~cm}^{-3}$. There is one more thing to remind you of at this point, namely, that because most of the energy resides in the high energy portion of a Planck curve, it does not matter much in an energy density measurement if you cut off most of the long wavelength side from your measurement. For example, a cutoff of the $2.7 \mathrm{~K}$ curve at $6 \mathrm{~mm}$ leaves nearly $98 \%$ of the total energy flux under the remainder of the curve. One of the numbers I shall refer to in this talk is the flux in a $2.7 \mathrm{~K}$ Planck curve between about 1.3 and $0.4 \mathrm{~mm}$; this number is about $4 \times 10^{-11} \mathrm{~W}$ $\mathrm{cm}^{-2}\left(\sim 0.1 \mathrm{eV} \mathrm{cm}^{-3}\right.$ energy density), or about $40 \%$ of the total flux from a $2.7 \mathrm{~K}$ blackbody background.

The earliest direct measurement of the background radiation on the short wavelength side of the $2.7 \mathrm{~K}$ Planck curve was made by Shivanandan et al. (1968). This experiment came in logical succession to the pioneer rocket infrared work of Harwit 
et al. (1966). By a nontrivial extension of the techniques developed for these earlier measurements at wavelengths of a few microns. Shivanandan et al. (1968) measured the background radiation at altitudes above $120 \mathrm{~km}$ integrated over the band from 0.4 to $1.3 \mathrm{~mm}$ wavelength. Their result was an astounding flux of $5 \times 10^{-9} \mathrm{~W} \mathrm{~cm}^{-2} \mathrm{sr}^{-1}$, with an estimated factor of two uncertainty. This value was approximately two orders of magnitude higher than the radiation intensity that would appear in this wavelength band from a $2.7 \mathrm{~K}$ isotropic blackbody source. Put in other terms, the result corresponded to radiation from a blackbody at a temperature of $8.3_{-1.3}^{+2.2} \mathrm{~K}$.

Some people believed the result, and some did not. Even if one did believe that the effect was not instrumental, there were, of course, explanations other than that it represented a measure of a universal background flux. As we shall see later, time appears to have been on the side of the skeptics.

Houck and Harwit (1969) repeated the experiment later that same year, with essentially the same radiometer, and obtained the same result. Somewhat later, they determined that their detector calibration had been in error, and they restated their results as just one-half the original values (Harwit et al., 1970). This was still a value about fifty times that expected from a $2.7 \mathrm{~K}$ blackbody background.

If interpreted on a galactic or cosmic scale, these results ran into trouble when confronting other data. In particular, the rocket experiment results were incompatible with the interstellar molecular data then available (Bortolot et al., 1969), unless one constructed an argument that concentrated the excess radiation in spectral lines.

Before going further, I should try to provide a fuller appreciation of the difficult nature of the submillimeter background experiments. The first problem is that, in general, measurement techniques in this wavelength region of the electromagnetic spectrum are more poorly developed than they are for several decades on either side. The employment of microwave detection techniques becomes impossible at wavelengths as short as a few millimeters while, on the other hand, there are no satisfactory photodetectors at wavelengths this long, and one is forced to use broadband bolometers with electromagnetic filtering; calibration sources are in a primitive state; etc. Fortunately for future experiments, several new developments promise to provide considerable aid in this region.

Secondly, since one is trying to measure radiation of very low intensity, over a broad wavelength band, the optical system must itself be a very weak emission source at these wavelengths, and this, in practice, means flying a liquid-helium-cooled device.

A third major problem is illustrated by Figure 2. Here, the brightness of a $2.7 \mathrm{~K}$ blackbody source is compared to that of a source at a temperature one hundred times larger. It is evident from the figure that if one attempts to make a measurement of flux intensity from a $2.7 \mathrm{~K}$ blackbody in the submillimeter wavelength range, with a $270 \mathrm{~K}$ blackbody radiating somewhere off to the side, one must build into one's instrument several orders of magnitude of off-axis rejection to this background source. Of course, this is exactly the situation faced in the present context, where the Earth and its lower atmosphere represent something approaching a $2 \pi$-sr source of blackbody radiation at $270 \mathrm{~K}$ or some slightly higher temperature. 
When totting up the difficulties, one must not forget that the entire instrument must be designed for operation in a rocket payload or, for some of the experiments I shall discuss, suspended from a balloon, and must withstand the considerable rigors of such a flight.

Figure 3 shows a schematic drawing of the telescope used by Harwit, Houck, and Shivanandan in the early rocket flights I have just discussed. The aperture of the system is about $16 \mathrm{~cm}$; the incoming radiation is modulated at the entrance to the

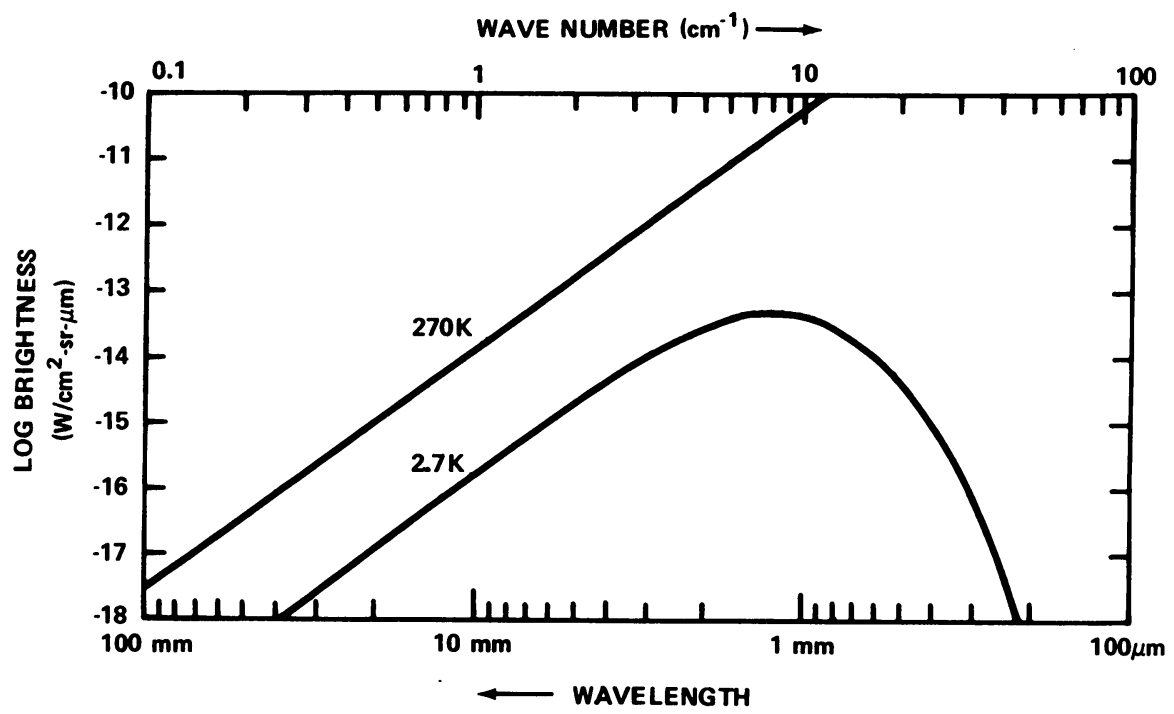

Fig. 2. Brightness curves for $2.7 \mathrm{~K}$ radiation and $270 \mathrm{~K}$ radiation.

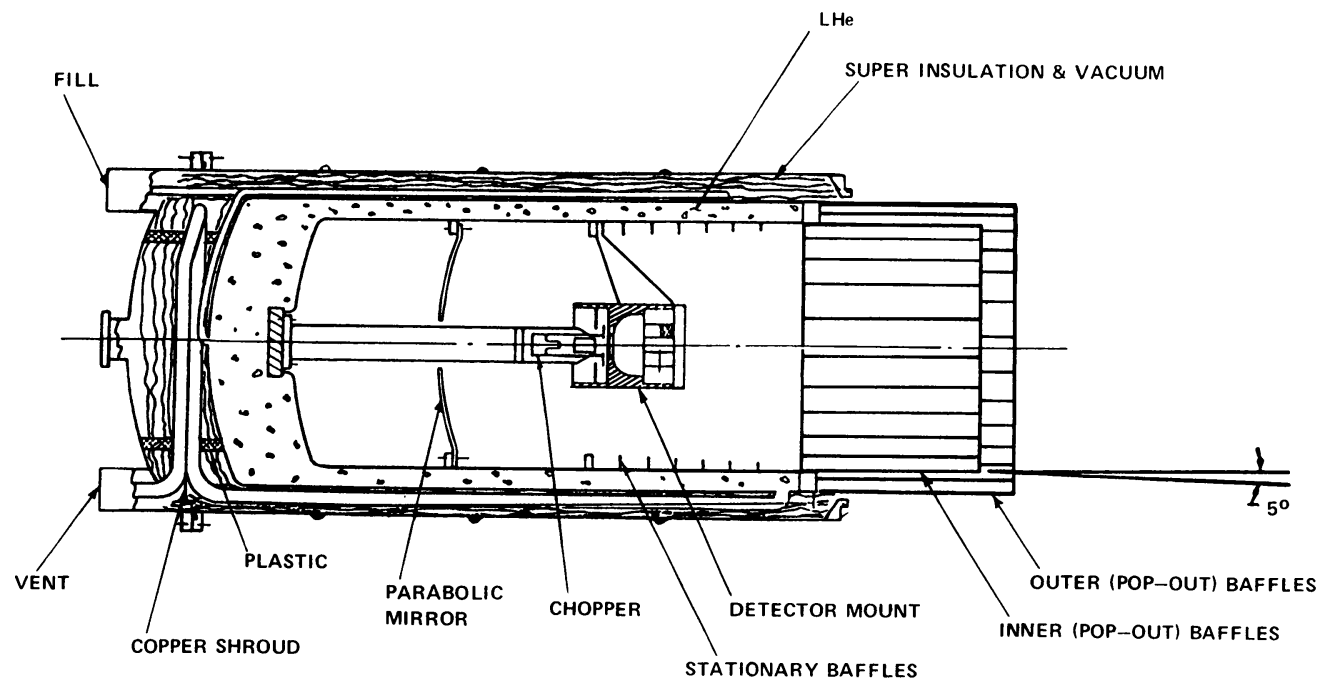

Fig. 3. Radiometer used in the early Cornell experiments (Harwit et al., 1969). 
detector cavity by a tuning fork chopper operating at $150 \mathrm{~Hz}$. Several detectors are housed inside the detector cavity; of these, only the InSb bolometer is of direct interest in the present discussion, since it was the one responding to radiation in the 1.3- to $0.4-\mathrm{mm}$ range. Note that the entire telescope is cooled with liquid helium.

In the autumn of the following year, 1969, Muehlner and Weiss (1970) of the Massachusetts Institute of Technology (MIT), conducted the first balloon-borne experiment to measure the submillimeter background. Here, the advantages of a long, soft ride, as compared to the harsh conditions of a rocket flight, are offset to some extent by the disadvantage of having to cope with the remaining atmosphere above the balloon altitude. In the wavelength range of interest, the principal atmospheric emission lines at balloon altitudes of about $40 \mathrm{~km}$ are from ozone, molecular oxygen, and water vapor. In this first MIT flight, there was considerable uncertainty in the correction for these emissions. In addition, the not inconsequential correction for the radiative contribution from various parts of the instrument itself could not be estimated with much confidence.

The radiometer used by Muehlner and Weiss in this experiment is shown in Figure 4. In contrast to the telescope employed by the Cornell-Naval Research Laboratory group, this instrument uses cone optics. Various filters are sequentially rotated into position during flight. Incoming radiation is modulated by a rotating chopper. The detector is an InSb bolometer, as in the Cornell experiments; the liquid helium bath is pumped by the ambient atmosphere, and is therefore at superfluid temperatures.

Muehlner and Weiss made measurements in three wavelength bands, as shown in Figure 5; their published results are given in Table II. Caroff and Petrosian (1971) later showed that additional allowance for the atmospheric radiative contribution to the signal should be made, so that these values could be reduced somewhat, but the data were still consistent with the interpretation of a strong source of excess radiation between 1.0 and $0.8 \mathrm{~mm}$.

The next development in this story was a new measurement of the submillimeter background radiation by the Cornell group in December, 1970. This time, the measurement over approximately the same spectral range as before yielded a somewhat smaller result, i.e., $\left(1.3_{-0.15}^{+0.10}\right) \times 10^{-9} \mathrm{~W} \mathrm{~cm}^{-2} \mathrm{sr}^{-1}$, still, however, a factor of twenty or so above that expected from a $2.7 \mathrm{~K}$ blackbody source. Moreover, the data indicated an isotropy within $\pm 10 \%$ over a galactic latitude range of about $20^{\circ}$.

In the spring of 1971, the Los Alamos Scientific Laboratory made a rocket measurement in this wavelength region. (A previous flight a year earlier had been unsuccessful because the rocket nose cone failed to separate from the payload.) The Los Alamos radiometer is shown schematically in Figure 6. This instrument has cone optics, tuning-fork choppers, gallium-doped germanium bolometers, and is precooled before launch to superfluid helium temperatures. There are three independent cone-chopperfilter-detector systems in this radiometer; of the two designed for measurements in the wavelength region under discussion, just one operated successfully. For this one, the system response is shown by the filter transmittance labeled 'LASL \# 1' in Figure 7. The result for this detector was consistent with the flux expected from a $2.7 \mathrm{~K}$ black- 


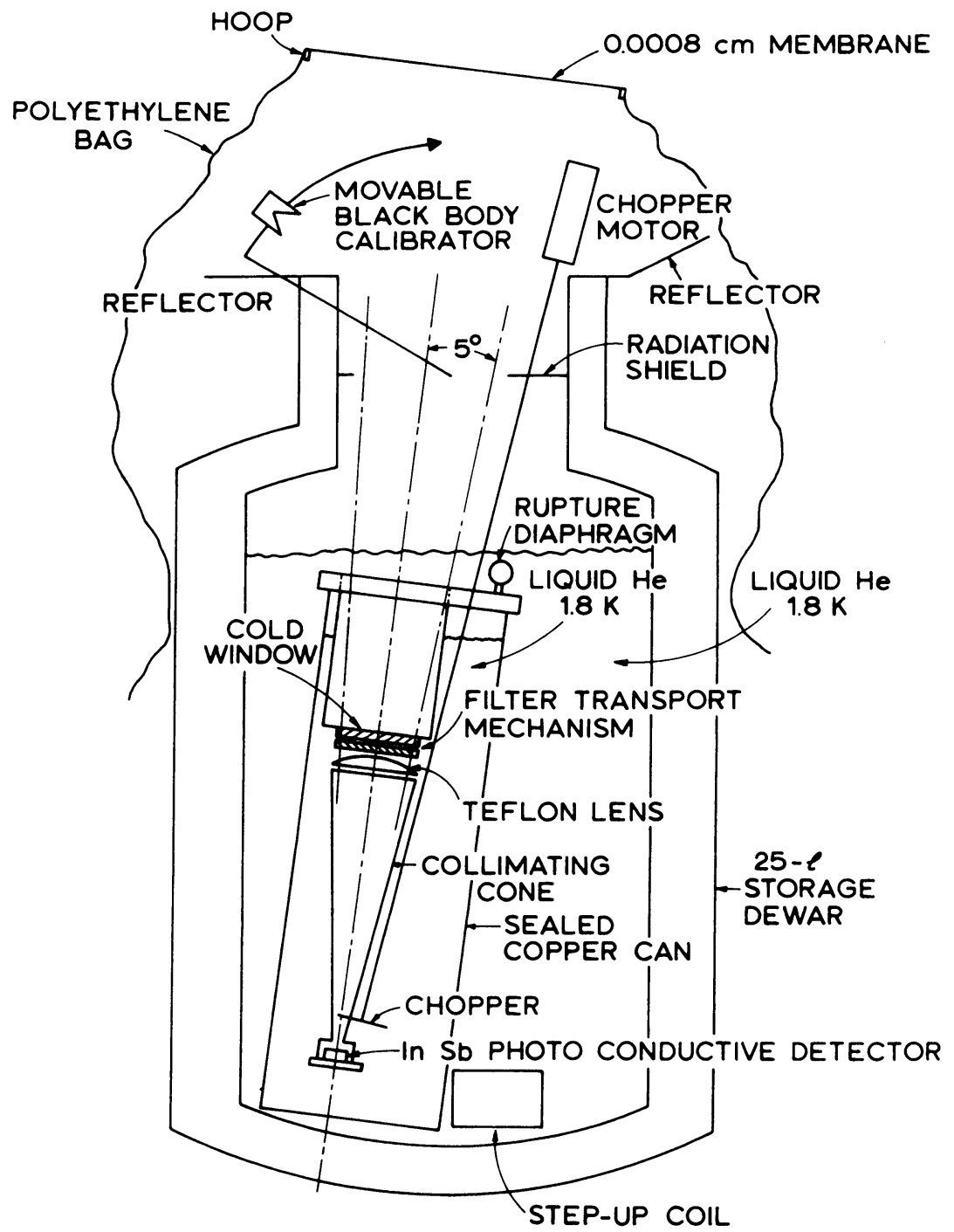

Fig. 4. Radiometer used in the first Massachusetts Institute of Techmology (MIT) experiment (Muehlner and Weiss, 1970).

body radiation; the measured flux was $99_{-8}^{+6} \times 10^{-11} \mathrm{~W} \mathrm{~cm}^{-2} \mathrm{sr}^{-1}$, and corresponded to an equivalent blackbody temperature of $3.1+1.0 \mathrm{~K}$. This result was in conflict with the data of Muehlner and Weiss. Figure 7 shows that the response curve of the two radiometers was very similar, but the Los Alamos experiment did not yield the high flux of the MIT experiment.

At this point, things stood as indicated in Figure 8. This figure shows the results of the first three Cornell rocket experiments, the first MIT balloon experiment, and the first Los Alamos rocket experiment. To help guide you through the figure, I must 
TABLE II

Fluxes and equivalent blackbody temperatures from 1969 MIT experiment

\begin{tabular}{llllll}
\hline $\begin{array}{l}\text { Spectral response } \\
(\mathrm{mm})\end{array}$ & \multicolumn{2}{l}{ Uncorrected results } & & \multicolumn{2}{c}{ 'Corrected' results } \\
\cline { 2 - 5 } & $T(\mathrm{~K})$ & $\begin{array}{c}\text { Minimum flux } \\
\left(\mathrm{W} \mathrm{cm} \mathrm{cm}^{-2} \mathrm{sr}^{-1}\right)\end{array}$ & & $T(\mathrm{~K})$ & $\begin{array}{l}\text { Minimum flux } \\
\left(\mathrm{W} \mathrm{cm}^{-2} \mathrm{sr}^{-1}\right)\end{array}$ \\
\hline $10-1.0$ & $7.4 \pm 0.2$ & $2.5 \times 10^{-9}$ & & 5.5 & $1.0 \times 10^{-9}$ \\
$10-0.8$ & $8.0 \pm 0.5$ & $8 \times 10^{-10}$ & & 7.0 & $6.4 \times 10^{-10}$ \\
$10-0.5$ & $4.7 \pm 0.3$ & $2 \times 10^{-10}$ & & 3.6 & $1.0 \times 10^{-10}$
\end{tabular}

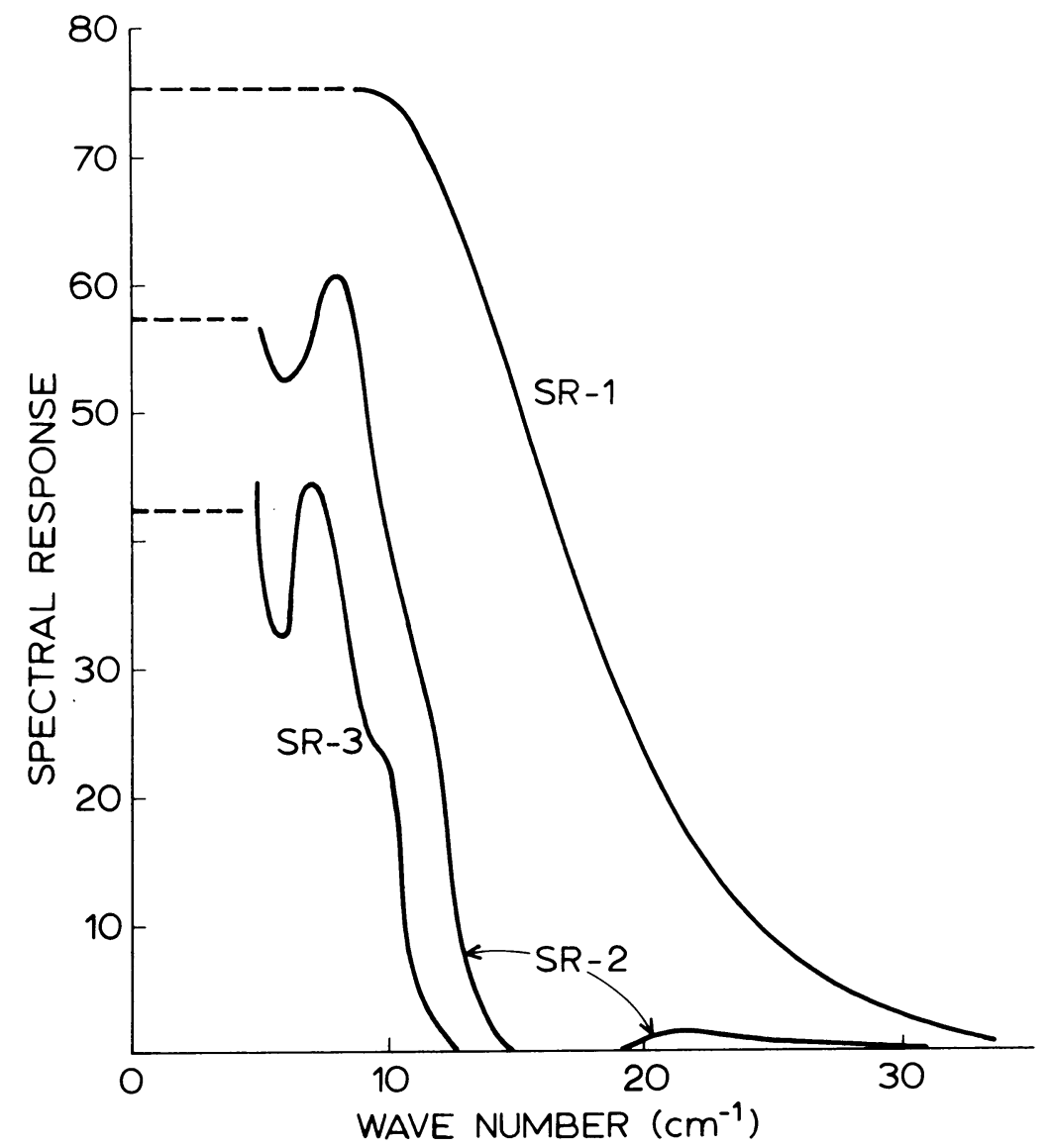

Fig. 5. Spectral responses of the radiometer in the first Massachusetts Institute of Technology (MIT) experiment (Muehlner and Weiss, 1970). 


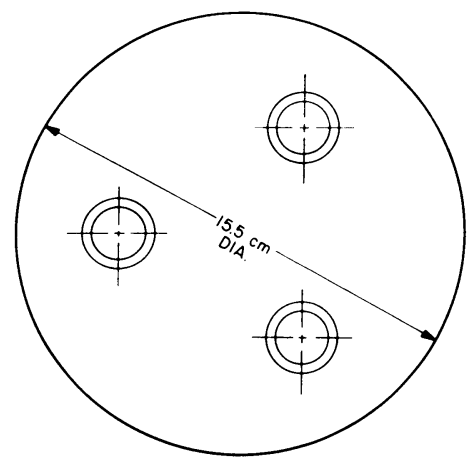

(b)

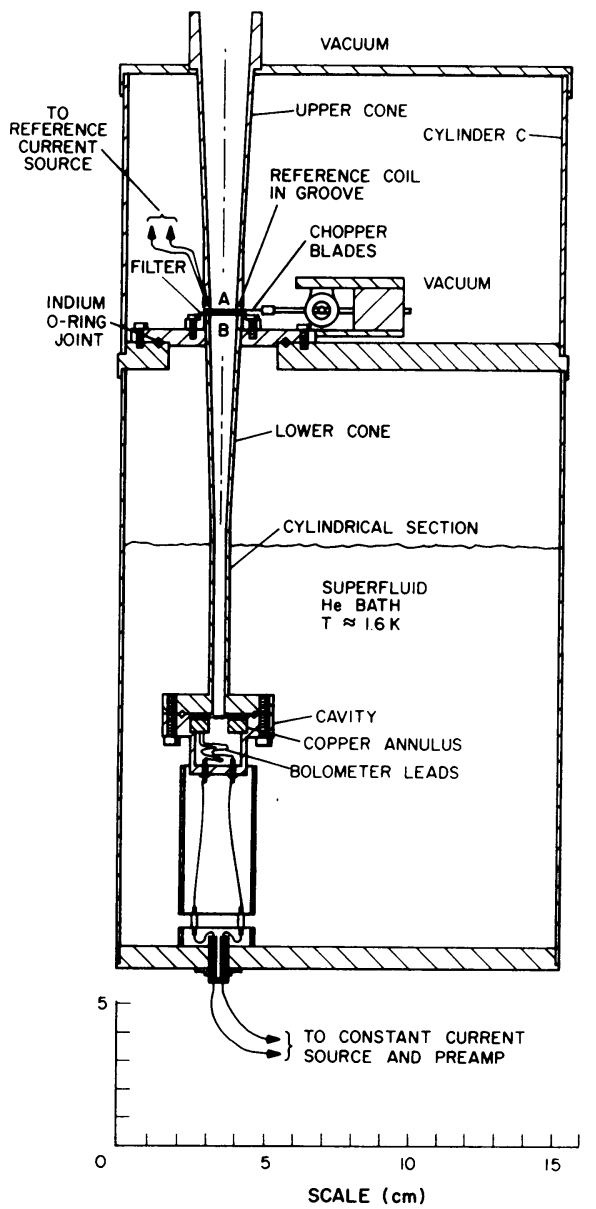

(a)

Fig. 6. Radiometer used in the Los Alamos Scientific Laboratory experiments. 

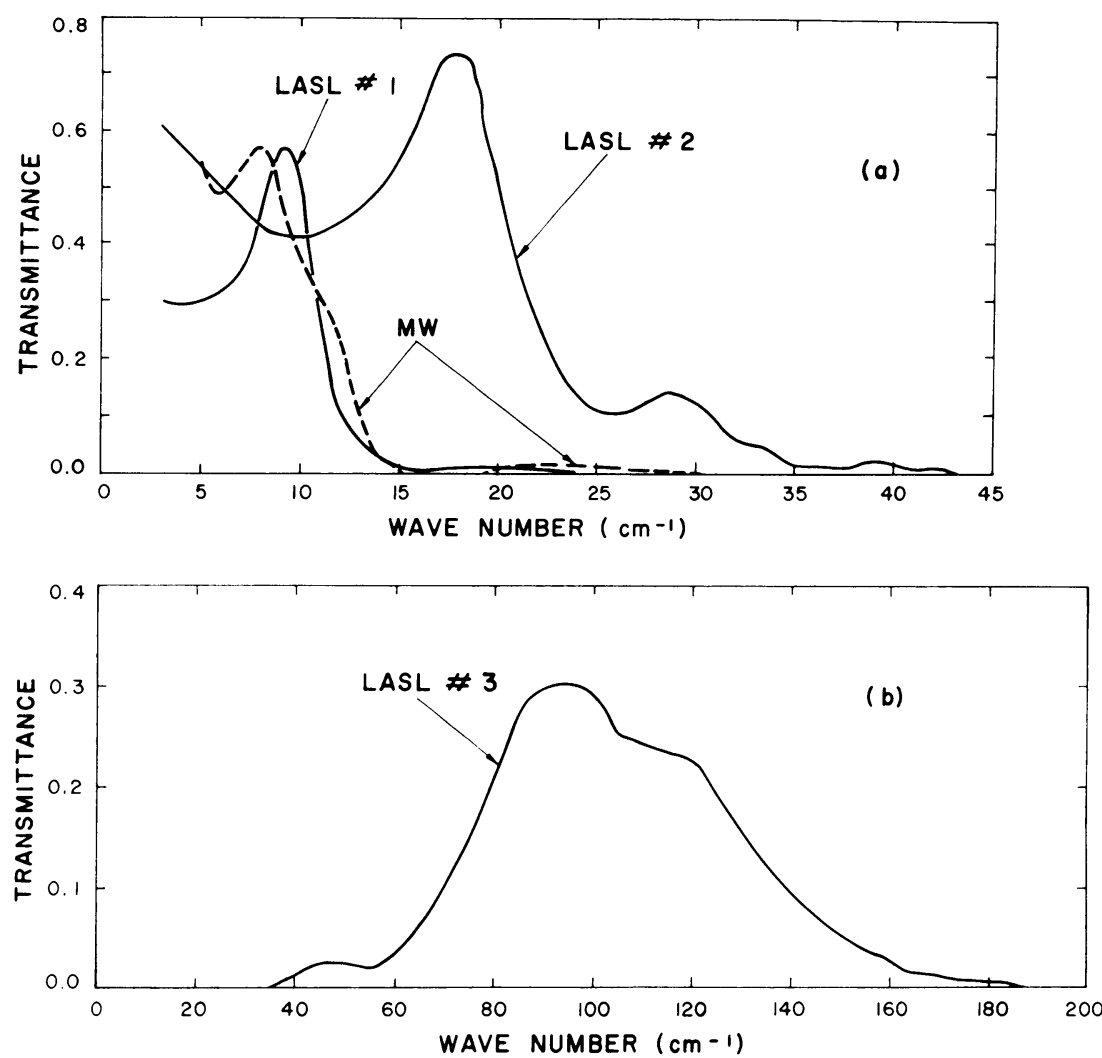

Fig. 7. Measured transmittances of filters used in the 1971 Los Alamos Scientific Laboratory (LASL) experiment. The curve labeled MW shows the response of the radiometer flown by Muehlner and Weiss (1970) in their 1969 experiment with filter SR-2 in position, normalized to the transmission of the LASL filter \# 1 at its peak.

first provide you with a little more background information. In a wide-band measurement of the type being discussed, it is customary to report the flux as that value required by the measurement if all the flux had been present in a line at the wavelength of maximum response of the radiometer. This reported flux is then really its minimum value; because the radiometer does not necessarily have a flat response within the passband, you can see that this method of reporting may underestimate the magnitude of the flux if a portion of it falls at some other wavelength within the passband. To be sure, one ought to know the response of one's radiometer as a function of wavelength and, accordingly, one can correct the data when displaying the results. The equivalent blackbody temperatures quoted for the various experiments $d o$ have the effects of the spectral shape of the radiometer response properly included.

In the case of the MIT experiment, since there were three overlapping passbands used, the results can, in principle, be refined further, and on this basis the allowable limits for the flux from this experiment lie within the indicated cross-hatched area in 


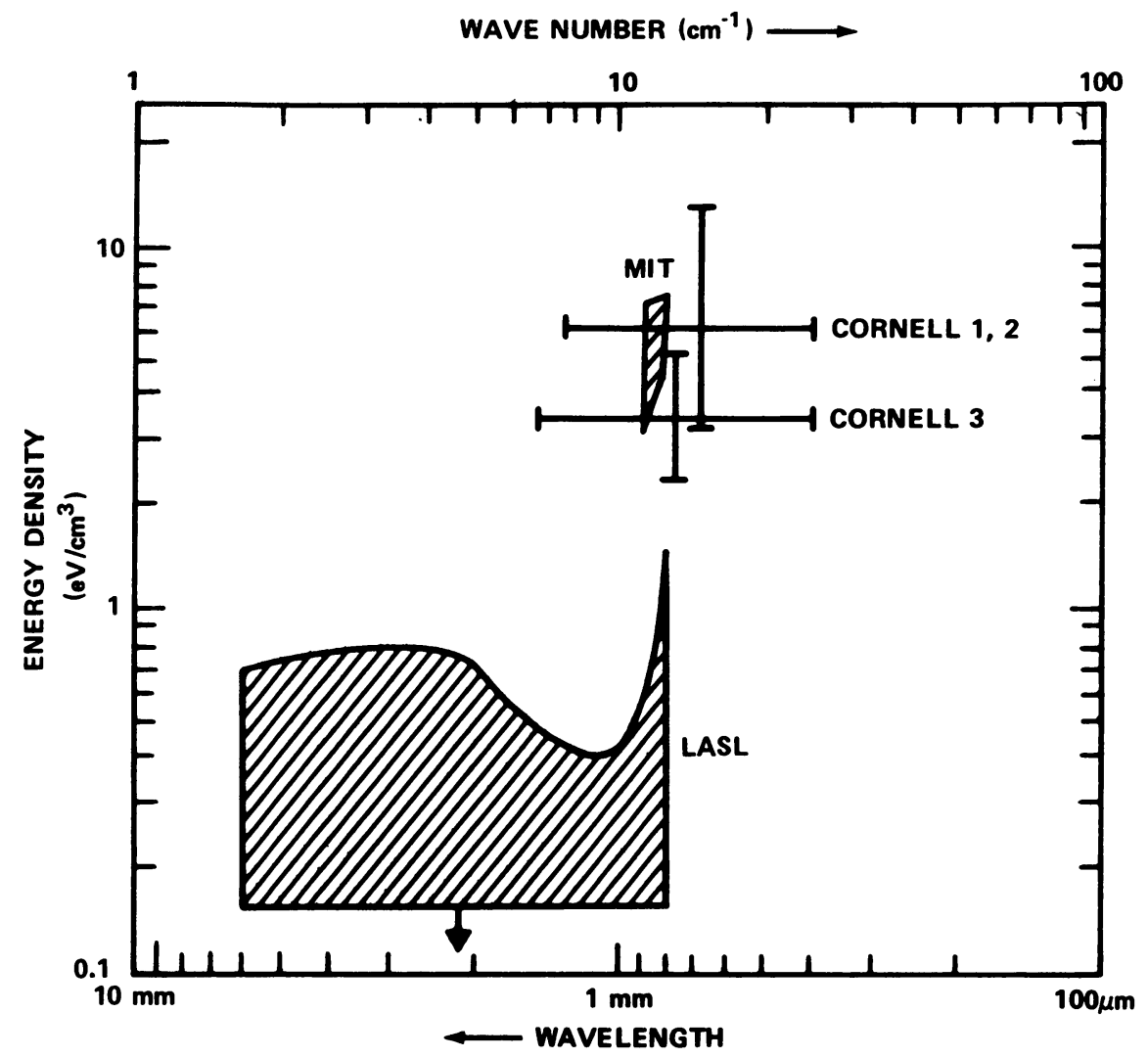

Fig. 8. Results of rocket and balloon measurements as of mid-1971.

the figure. The analysis actually shown in this case is due to Caroff and Petrosian(1971). Because I do not know accurately the spectral response of the Cornell radiometer, I have simply indicated the measured value over the entire passband, but clearly the curve must turn upward on each end. The Los Alamos result shown has, folded into it, the spectral response of the radiometer.

It is clear from the figure that (a) the Los Alamos results and the MIT results are mutually exclusive, (b) the Cornell and MIT results are compatible with each other, and (c) the Los Alamos and Cornell results are not incompatible if one wishes to assume that the intense radiation seen by Cornell lies at relatively short wavelengths.

Fortunately, everyone made more measurements. In these new experiments, the large fluxes seen previously by both the Cornell group and the MIT group were not observed. Equally important, at least from my point of view, large fluxes were not observed in the new Los Alamos experiment.

Table III provides a tabulation of the results from the September 29, 1971 balloon flight of the MIT group (Muehlner and Weiss, 1973a). This group also conducted a balloon experiment in June of that same year (Muehlner and Weiss, 1973a), and an- 
TABLE III

Corrected fluxes and equivalent blackbody temperatures from September 1971 MIT experiment

\begin{tabular}{llc}
$\begin{array}{l}\text { Normalized box spectral } \\
\text { response }(\mathrm{mm})\end{array}$ & $T(\mathrm{~K})$ & Minimum flux $\left(\mathrm{W} \mathrm{cm}^{-2} \mathrm{sr}^{-1}\right)$ \\
\hline $10-1.8$ & $2.7_{-0.6}^{+0.4}$ & $(3.3 \pm 1.7) \times 10^{-11}$ \\
$10-1.3$ & $2.8 \pm 0.2$ & $(6.0 \pm 1.5) \times 10^{-11}$ \\
$10-1.3$ & $2.8 \pm 0.2$ & $(5.6 \pm 1.5) \times 10^{-11}$ \\
$10-0.9$ & $\leqslant 2.7$ & $\leqslant 6.2 \times 10^{-11}$ \\
$10-0.5$ & $\leqslant 3.4$ & $\leqslant 2.3 \times 10^{-10}$ \\
\hline
\end{tabular}

other experiment in October, 1972 (Muehlner and Weiss, 1973b), whose results are not shown here, but which yielded results in substantial agreement with those from the September 1971 flight. The blackbody temperatures deduced are in excellent agreement with $2.7 \mathrm{~K}$. In this experiment, atmospheric corrections were made on the basis of the signal from the radiometer vs its zenith angle. Great care was taken in the design of the apparatus to shield the radiometer from hot sources at large angles to its optical axis.

On 17 May, 1972, the Los Alamos group conducted another rocket experiment (Williamson et al., 1973). The radiometer used was essentially identical in design to that used in the first experiment; the response of the radiometer is given by the filter transmittances shown in Figure 9. Several difficulties were encountered in the flight, and as a result, the error bars on the results were quite large. The experiment did, however, confirm the absence of a large background such as that observed in the earlier Cornell experiments. The data from the radiometer section sensitive to radiation over the widest spectral range, from about 0.3 to $6 \mathrm{~mm}$, yielded an equivalent blackbody temperature of $3.8_{-3.8}^{+1.0} \mathrm{~K}$, consistent with a $2.7 \mathrm{~K}$ blackbody background, but consistent with a lot of other temperatures too, including zero.

I finally arrive at what is, hopefully, the coup de grace to the intense submillimeter background flux phenomenon. On 18 July, 1972, the Cornell group made another rocket measurement of the background flux in the 0.4- to 1.3-mm range (Houck et al., 1972). For this experiment, their radiometer was modified to be less sensitive to various effects that might produce signal contamination; this was done primarily by passing the observed radiation through additional field and aperture stops, and by reducing possible radiofrequency interference.

The data from this experiment yielded a background flux of $(1.6 \pm 2.0) \times 10^{-10} \mathrm{~W}$ $\mathrm{cm}^{-2} \mathrm{sr}^{-1}$. As you will remember from earlier in this paper, the expected flux from a $2.7 \mathrm{~K}$ blackbody background over this range is $0.4 \times 10^{-10} \mathrm{~W} \mathrm{~cm}^{-2} \mathrm{sr}^{-1}$. It is important to note that this result, while consistent with a $2.7 \mathrm{~K}$ background, is also consistent with some other temperatures, including zero.

I think that the scare is over; in my own mind, there is not much doubt that the submillimeter cosmic background has been shown to be low. Additional substantiation has come from ground-based and aircraft-based searches for extra-atmospheric 
emission lines. The most recent of these results in general preclude an intensity greater than about $10^{-9} \mathrm{~W} \mathrm{~cm}^{-2} \mathrm{sr}^{-1}$ in the wavelength range from 0.4 to $1 \mathrm{~mm}$ (Nolt et al., 1972; Beckman et al., 1972).

I do not want to speculate on what might have been the source of the earlier high measurements, except to reiterate my previous comment that these are difficult ex-

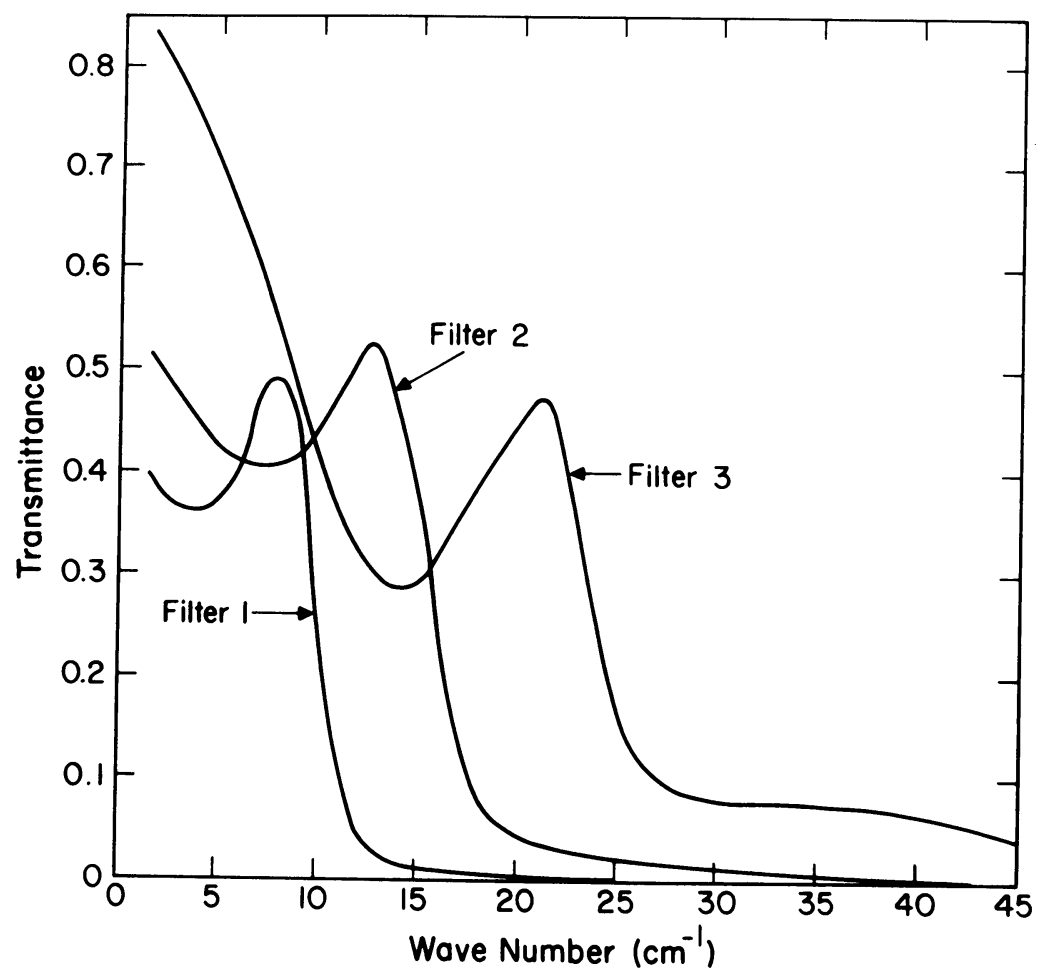

Fig. 9. Measured transmittances of filters used in the 1972 Los Alamos Scientific Laboratory experiment.

periments. The state of the art has certainly been advanced by this work, and has laid a part of the groundwork for the next generation of experiments, namely, the detailed spectral measurements of the background radiation in this submillimeter range.

In this connection, it is important to note well what hasn't been shown yet. There is a tendency to say that the recent results lend support to the notion of a $2.7 \mathrm{~K}$ background; I've made that statement myself. This is true in the sense that these results are compatible with a $2.7 \mathrm{~K}$ background, while the earlier results weren't. But, in fact, the measurements give very little quantitative information about the submillimeter background radiation. At wavelengths less than $1 \mathrm{~mm}$, all one has from these measurements is upper limits to the flux. At slightly longer wavelengths, say between 1 and $2 \mathrm{~mm}$, the MIT results would appear to be somewhat more informative. These results need to be verified by independent measurements, of course; even if correct they provide no facts about the spectral shape in this wavelength region. 
At still longer wavelengths, the ground starts to firm up, although there is still no detailed knowledge about the shape of the spectrum in the millimeter and centimeter range.

I believe that most of the excitement lies ahead, as attempts are made to fly interferometers and, possibly, tunable detectors, first on balloon platforms and then on spacecraft.

\section{References}

Apparao, M. V. Krishna: 1968, Nature 219, 709.

Beckman, J. E., Ade, P. A. R., Huizinga, J. S., Robson, E. I., Vickers, D. G., and Harries, J. E. : 1972 , Nature 237, 154.

Bortolot, V. J., Jr., Clauser, J. F., and Thaddeus, P.: 1969, Phys. Rev. Letters 22, 307.

Boyton, P. E., Stokes, R. A., and Wilkinson, D. T.: 1968, Phys. Rev. Letters 21, 462.

Caroff, L. J. and Petrosian, V.: 1971, Nature 231, 378.

Cowsik, R. and Price, P. B.: 1971, Phys. Today 24, 30.

Dicke, R. H., Peebles, P. J. E., Roll, P. G., and Wilkinson, D. T.: 1965, Astrophys. J. 142, 414.

Harwit, M., Houck, J. R., and Fuhrmann, K.: 1969, Appl. Opt. 8, 473.

Harwit, M., Houck, J. R., and Wagoner, R. V.: 1970, Nature 228, 451.

Harwit, M., McNutt, D. P., Shivanandan, K., and Zajac, B. J. : 1966, Astron. J. 71, 1026.

Houck, J. R. and Harwit, M.: 1969, Astrophys. J. 157, L45.

Houck, J. R., Soifer, B. T., Harwit, M., and Pipher, J. L.: 1972, Astrophys. J. 178, L29.

Millea, M. F., McColl, M., Pedersen, R. J., and Vernon, F. L., Jr.: 1971, Phys. Rev. Letters $26,919$.

Muehlner, D. and Weiss, R.: 1970, Phys. Rev. Letters 24, 742.

Muehlner, D. and Weiss, R.: 1973a, Phys. Rev. D7, 326.

Muehlner, D. and Weiss, R.: 1973b, Phys. Rev. Letters 30, 757.

Nolt, I. G., Radostitz, J. V., and Donnelly, R. J.: 1972, Nature 236, 444.

Penzias, A. A. and Wilson, R. W.: 1965, Astrophys. J. 142, 419.

Shivanandan, K., Houck, J. R., and Harwit, M. O.: 1968, Phys. Rev. Letters 21, 1460.

Thaddeus, P.: 1972, Ann. Rev. Astron. Astrophys. 10, 305 (see for a recent review of these results).

Williamson, K. D., Blair, A. G., Catlin, L. L., Hiebert, R. D., Loyd, E. G., and Romero, H. V.: 1973, Nature 241, 79.

\section{DISCUSSION}

Rees: At the recent IAU General Assembly in Sydney, Zuckerman reported some new work on interstellar formaldehyde, which showed (if I recall correctly) that the temperature at $\sim 2 \mathrm{~mm}$ could not differ substantially from the temperature at $\sim 6 \mathrm{~cm}$.

Zel'dovich: How well is the spectrum of the microwave background known to follow the Planck law at centimetre wavelengths?

Blair: Of nearly 20 ground-based measurements made to date, between 73.5 and $0.33 \mathrm{~cm}$, the errors on the measured temperature range between 1 or 2 tenths of a deg to about $1 \mathrm{deg}$. The latest optical absorption measurement at $2.64 \mathrm{~mm}$ has an error in temperature of only $0.10 \mathrm{~K}$. 